\title{
Validity of self-reported adult secondhand smoke exposure
}

\author{
Judith J Prochaska, ${ }^{1}$ William Grossman, ${ }^{2}$ Kelly C Young-Wolff, ${ }^{1}$ Neal L Benowitz ${ }^{3}$
}

${ }^{1}$ Department of Medicine, Stanford University, Stanford Prevention Research Center Stanford, California, USA ${ }^{2}$ Department of Medicine, Division of Cardiology, University of California, San Francisco, San Francisco, California, USA

${ }^{3}$ Departments of Medicine and Bioengineering and

Therapeutic Sciences, Division of Clinical Pharmacology and Experimental Therapeutics, University of California, San Francisco, San Francisco, California, USA

\section{Correspondence to} Dr Judith J Prochaska, School of Medicine, Stanford Prevention Research Center, Stanford University, Medical School Office Building, X316, 1265 Welch Road, Stanford, CA 94305-5411, USA; JPro@Stanford.edu.

Received 28 May 2013 Accepted 8 August 2013 Published Online First 30 August 2013

\section{CrossMark}

To cite: Prochaska JJ, Grossman W, Young-

Wolff KC, et al. Tob Control 2015:24:48-53.

\begin{abstract}
Objectives Exposure of adults to secondhand smoke (SHS) has immediate adverse effects on the cardiovascular system and causes coronary heart disease. The current study evaluated brief self-report screening measures for accurately identifying adult cardiology patients with clinically significant levels of SHS exposure in need of intervention.
\end{abstract}

Design and setting $A$ cross-sectional study conducted in a university-affiliated cardiology clinic and cardiology inpatient service.

Patients Participants were 118 non-smoking patients ( $59 \%$ male, mean age $=63.6$ years, $S D=16.8$ ) seeking cardiology services.

Main outcome measures Serum cotinine levels and self-reported SHS exposure in the past $24 \mathrm{~h}$ and 7 days on 13 adult secondhand exposure to smoke (ASHES) items.

Results A single item assessment of SHS exposure in one's own home in the past 7 days was significantly correlated with serum cotinine levels $(r=0.41, p<0.001)$ with sensitivity $\geq 75 \%$, specificity $>85 \%$ and correct classification rates $>85 \%$ at cotinine cut-off points of $>0.215$ and $>0.80 \mathrm{ng} / \mathrm{mL}$. The item outperformed multiitem scales, an assessment of home smoking rules, and SHS exposure assessed in other residential areas, automobiles and public settings. The sample was less accurate at self-reporting lower levels of SHS exposure (cotinine $0.05-0.215 \mathrm{ng} / \mathrm{mL}$ ).

Conclusions The single item ASHES-7d Home screener is brief, assesses recent SHS exposure over a week's time, and yielded the optimal balance of sensitivity and specificity. The current findings support use of the ASHES$7 \mathrm{~d}$ Home screener to detect SHS exposure and can be easily incorporated into assessment of other major vital signs in cardiology.

\section{INTRODUCTION}

Exposure of adults to secondhand smoke (SHS) has immediate adverse effects on the cardiovascular system. ${ }^{1}$ A 2009 report of the Institutes of Medicine concluded that non-smokers exposed to SHS have a $45 \%$ increased risk of developing major coronary heart disease. ${ }^{2}$ Of the 53000 nonsmoker deaths due to SHS exposure in the USA each year, three in four are cardiovascular-related.

Much of the acute cardiovascular risk of smoking and SHS is due to activation of platelets and impairment of endothelial function, which result in vascular thrombosis and restricted vasodilation, which in turn result in reduced coronary or cerebral blood flow. ${ }^{1}{ }^{3}$ Smoking bans have helped protect the public from involuntary SHS exposure in workplaces, restaurants, bars and public places, ${ }^{4-6}$ and adoption of comprehensive smoke-free legislation is associated with a $15 \%-17 \%$ decline in acute coronary events and stroke. ${ }^{7-9}$

While SHS exposure in the USA has decreased substantially in recent decades, approximately $40 \%$ of non-smokers continue to be exposed, with higher rates of exposure among individuals of lower income, with less education, who are older, and who identify as African American or non-Hispanic Caucasian compared with Asian or Hispanic. ${ }^{10}$ Of particular concern are SHS exposures in residential settings and automobiles, areas that are largely unlegislated. Provider counselling on tobacco use doubles the likelihood of smokers quitting ${ }^{11}$ and may similarly prove useful in reducing patients' exposure to SHS in residential locations and cars. An initial step for prompting provider counselling on SHS is identification of patients at risk.

Biomarkers of tobacco use and SHS exposure include cotinine, a major metabolite of nicotine detected in the blood, urine or saliva for up to $72 \mathrm{~h},{ }^{12}$ and NNAL (4-(methylnitrosamino)-1(3-pyridyl)-1-butanol), a metabolite of the highly carcinogenic tobacco-specific nitrosamine NNK (4-(methylnitrosamino)-1-(3-pyridyl)-1-butanone) detectible in urine for up to 12 weeks. ${ }^{13-15}$ Examination of serum cotinine in 965 adult patients admitted to an urban, public hospital indicated $40 \%$ of patients were active smokers (cotinine $\geq 14 \mathrm{ng}$ / $\mathrm{mL}), 14 \%$ were recent smokers or heavily exposed to SHS $(0.5-13.9 \mathrm{ng} / \mathrm{mL})$, and $25 \%$ had low level SHS exposure $(0.05-0.49 \mathrm{ng} / \mathrm{mL}) .{ }^{16}$ In the Third National Health and Nutrition Examination Survey (NHANES III), both lower $(0.05-0.215 \mathrm{ng} / \mathrm{mL})$ and higher $(>0.215 \mathrm{ng} / \mathrm{mL})$ cotinine levels were associated with elevations in fibrinogen and homocysteine, biomarkers of heart disease. ${ }^{17}$ The analysis of Whincup and colleagues of prospective data from the British regional heart study found that heavy SHS exposure, defined as serum cotinine between 0.80 and $14 \mathrm{ng} / \mathrm{mL}$, carried a risk of major coronary heart disease comparable with light active smoking (1-9 cigarettes/day). ${ }^{18}$

In clinical practice, assessments of cotinine or other biomarkers of tobacco exposure are underused, often unavailable outside of specialty labs, costly and time-delayed for obtaining results. Self-report measures of SHS exposure have been used in epidemiological investigations and validated against cotinine. ${ }^{19}{ }^{20}$ Most studies have focused on parent-reported youth or neonate exposure to SHS, with the strength of association varying considerably depending on the reference population and assessment measure. Sensitivity of the measures, defined as the proportion of SHS-exposed participants correctly identified, has ranged from 30\% to 
$85 \%$. Specificity, the proportion of SHS-unexposed participants correctly identified, has ranged from $52 \%$ to $97 \%$. $^{19}$

Screening measures for detecting SHS exposure among adult patients have not been evaluated for correct classification, and a group of particular relevance is patients at risk for heart disease. Adults may have greater difficulty reporting on their own SHS exposure due to encountering a wider range of environments and perhaps less heightened awareness when SHS exposures do not involve their children. Assessment among patients at risk for heart disease is of specific interest due to greater vulnerability to SHS's cardiotoxic effects. Further, demand characteristics with this patient group may lead to under-reporting SHS exposure, a known toxin, when interviewed in a medical setting. By comparison, in the tobacco treatment literature, misreporting of primary smoking among patients with heart disease has led to recommendations for biochemical verification. ${ }^{21}$ The current study, conducted with patients seen in university-affiliated cardiology clinics and an inpatient cardiology service, aimed to evaluate the utility of brief self-report SHS screening items for accurately identifying adult patients with clinically significant levels of SHS exposure in need of intervention.

\section{METHODS}

\section{Participants and procedures}

The sample included patients of the University of California, San Francisco (UCSF) cardiology outpatient service, an insured patient population, and patients hospitalised in cardiology at San Francisco General Hospital, a largely uninsured patient population. With informed consent and approval from the UCSF IRB, patients who completed brief measures of SHS exposure and agreed to a voluntary cotinine test were included in the study. For the outpatients, the cotinine test was in addition to their scheduled blood draw. For the inpatients, their hospital admission leftover blood samples were obtained and assayed for cotinine, thereby providing an indication of SHS prior to hospitalisation. Active current smokers and non-English speakers were excluded. Data were collected between March 2010 and July 2011.

\section{Measures}

ASHES items

We evaluated 13 self-report items of adult SHS exposure (figure 1). The items were adapted from the 2009 Social Climate Survey of Tobacco Control (http://www.socialclimate.org). Twelve items asked about exposures to SHS in two reference periods (past 24 h or past 7 days) in six settings: (a) personal residence, (b) friends' residence, (c) relatives' residence, (d) in automobiles, (e) in a public area and (f) somewhere else. Hospitalised patients were asked about exposures in the $24 \mathrm{~h}$ and 7 days prior to hospitalisation. Each item was coded as exposed (1) or unexposed $(0)$. The items were evaluated individually and as multiitem scales for each reference period. For the scales, respondents endorsing SHS exposure in any one of the assessed settings were considered SHS-exposed. The thirteenth item asked about home smoking rules with response options of (a) no one is allowed to smoke anywhere, (b) smoking is permitted in some places at some times and (c) smoking is permitted anywhere.

\section{Cotinine validation}

Serum cotinine samples were analysed with liquid chromatographymass spectrometry, with a limit of quantitation of $0.02 \mathrm{ng} / \mathrm{mL} .^{22}$ Active smoking status was determined using the serum cotinine cutpoint of $14 \mathrm{ng} / \mathrm{mL}$, which provides 96\%-97\% sensitivity and $99 \%-100 \%$ specificity. ${ }^{23}$ Because there is no established cut-point for defining heavy SHS exposure versus light SHS exposure, we explored three different cut-off points for categorising serum cotinine: $0.05,0.215$ and $0.80 \mathrm{ng} / \mathrm{mL}$ based on prior evidence of cardiovascular health effects at these levels. ${ }^{17} 18$

\section{Demographic variables}

From medical records, we obtained information on patients' age, gender, marital status and ethnicity with coding limited to Hispanic versus non-Hispanic.

\section{Statistical analyses}

To describe the sample, we calculated mean age and percentiles for gender, Hispanic ethnicity, marital status and recruitment site. We examined demographic differences in cotinine levels using the Mann-Whitney U test for two samples (ie, gender, ethnicity, recruitment site) and the Kruskal-Wallis 1-way ANOVA for $\mathrm{k}$ samples (ie, marital status, age group), given our anticipation that the cotinine values would be non-normally distributed. We calculated for each screening item and the three cotinine cut-off points the percentage of participants with SHS exposure. To test the strength of association between the adult secondhand exposure to smoke (ASHES) self-report items and measured cotinine levels, we ran point-biserial correlations. Items that significantly correlated with cotinine were further analysed for consideration as individual screening items and as part of multi-item scales. Cronbach $\alpha$ values were calculated to examine internal consistency of the items for the two different reference periods ( $24 \mathrm{~h}$ and past 7 days). To estimate the relative clinical utility of the brief screening items and scales as part of cardiovascular medical care, the proportion of study participants with detected SHS exposure (defined as serum cotinine between $0.05-14 \mathrm{ng} / \mathrm{mL}, \quad 0.215-14 \mathrm{ng} / \mathrm{mL}$ and $0.80-14 \mathrm{ng} / \mathrm{mL}$ ) was determined and compared with the proportion of patients SHS-exposed based on responses to the ASHES items. We calculated sensitivity (ie, the proportion of SHS-exposed participants based on cotinine level correctly identified as such by the ASHES items) and specificity (ie, the proportion of SHS-unexposed participants based on cotinine level correctly identified as unexposed by the ASHES items). Prioritisation was given to sensitivity over specificity given the low clinical implications of falsely screening someone as SHS-exposed versus missing a clinical opportunity to counsel someone who is exposed but undetected. Last, we calculated correct classification of the measures (ie, the proportion correctly identified as exposed and unexposed).

\section{RESULTS}

\section{Sample description}

Of 126 patients who completed the ASHES survey and provided a cotinine sample, eight had levels $>14 \mathrm{ng} / \mathrm{mL}$ indicative of active smoking and were excluded from further analyses. The 118 non-smoking participants had a mean age of 63.6 years $(\mathrm{SD}=16.8$, range 24-93) and were 59\% male; $45 \%$ were married, $31 \%$ single, $13 \%$ widowed and $11 \%$ divorced. The sample was 13\% Hispanic and most were outpatients (91\%). Cotinine levels did not differ significantly by age or Hispanic ethnicity but did by recruitment site, marital status and gender with higher levels among hospitalised patients, divorced individuals and men (all $\mathrm{p}<0.001$ in non-parametric group comparisons).

Of the full sample, $28.8 \%$ had a cotinine level $>0.05 \mathrm{ng} / \mathrm{mL}$, $10.2 \%>0.215 \mathrm{ng} / \mathrm{mL}$ and $5.9 \%>0.80 \mathrm{ng} / \mathrm{mL}$. Figure 2 shows the per cent reporting SHS exposure on the ASHES items. More participants reported SHS exposure in the past 7 days 
Secondhand smoke is a mixture of the smoke exhaled by smokers and the smoke that comes off the burning end of a cigarette.

\begin{tabular}{|c|c|}
\hline \multicolumn{2}{|c|}{ In the past 7 days, were you exposed to secondhand smoke: } \\
\hline 1. where you live? & [ ] no [ ] yes \\
\hline 2. at a friend's home? & [ ] no [ ] yes \\
\hline 3. at a relative's home? & [ ] no [ ] yes \\
\hline 4. in a car? & [ ] no [ ] yes \\
\hline 5. in a public area? & [ ] no [ ] yes \\
\hline 6. somewhere else? & [ ] no [ ] yes, where? \\
\hline \multicolumn{2}{|c|}{ In the past 24 hours, were you exposed to secondhand smoke: } \\
\hline 7. where you live? & [ ] no [ ] yes \\
\hline 8. at a friend's home? & [ ] no [ ] yes \\
\hline 9. at a relative's home? & [ ] no [ ] yes \\
\hline 10. in a car? & [ ] no [ ] yes \\
\hline 11. in a public area? & [ ] no [ ] yes \\
\hline 12. somewhere else? & [ ] no [ ] yes, where? \\
\hline
\end{tabular}

13. What rule applies to smoking where you live?

[ ] No one is allowed to smoke anywhere

[ ] Smoking is permitted in some places at some times

[ ] Smoking is permitted anywhere

Figure 1 Adult secondhand exposure to smoke items.

than in the past $24 \mathrm{~h}$. SHS in public places was most commonly reported: $60 \%$ of participants exposed in the past 7 days. More than a third (past $24 \mathrm{~h}$ ) and two-thirds (past 7 days) of participants reported SHS exposure in any setting. Regarding home smoking rules, $72.9 \%$ reported smoking was not permitted in their home at anytime, $19.5 \%$ reported smoking is permitted in some areas of their home at some times and 7.6\% said smoking is permitted anywhere. Four of the 86 participants who stated smoking is not permitted in their home reported SHS exposure in their own residence in the past 7 days.

\section{Correlations}

For both the past 24-h and 7-day reference periods, reported SHS exposure in the residence of one's own or that of family or friends correlated significantly with cotinine levels (table 1), as did the item assessing home smoking rules, $r=0.38, p<0.001$.
For automobile SHS exposure, only the past 24-h item correlated significantly with cotinine levels. Reports of SHS exposure in public places and other areas did not correlate significantly with cotinine levels. Sum score composites, calculated for the items that were significant, had reasonable internal consistency and also correlated significantly with cotinine levels: past 7-day residential exposure (three items summing reported SHS exposure in the residence of one's own, of friends and of relatives, range $0-3$, Cronbach $\alpha=0.66, \mathrm{r}=0.53, \mathrm{p}<0.001$ ) and past $24-\mathrm{h}$ residential and automobile exposure (four items summing the three residential items with automobile SHS exposure, range $0-4$, Cronbach $\alpha=0.69, \mathrm{r}=0.46, \mathrm{p}<0.001$ ).

\section{Classification rates}

Table 2 summarises the sensitivity, specificity and correct classification rates for the eight retained individual items and the two 


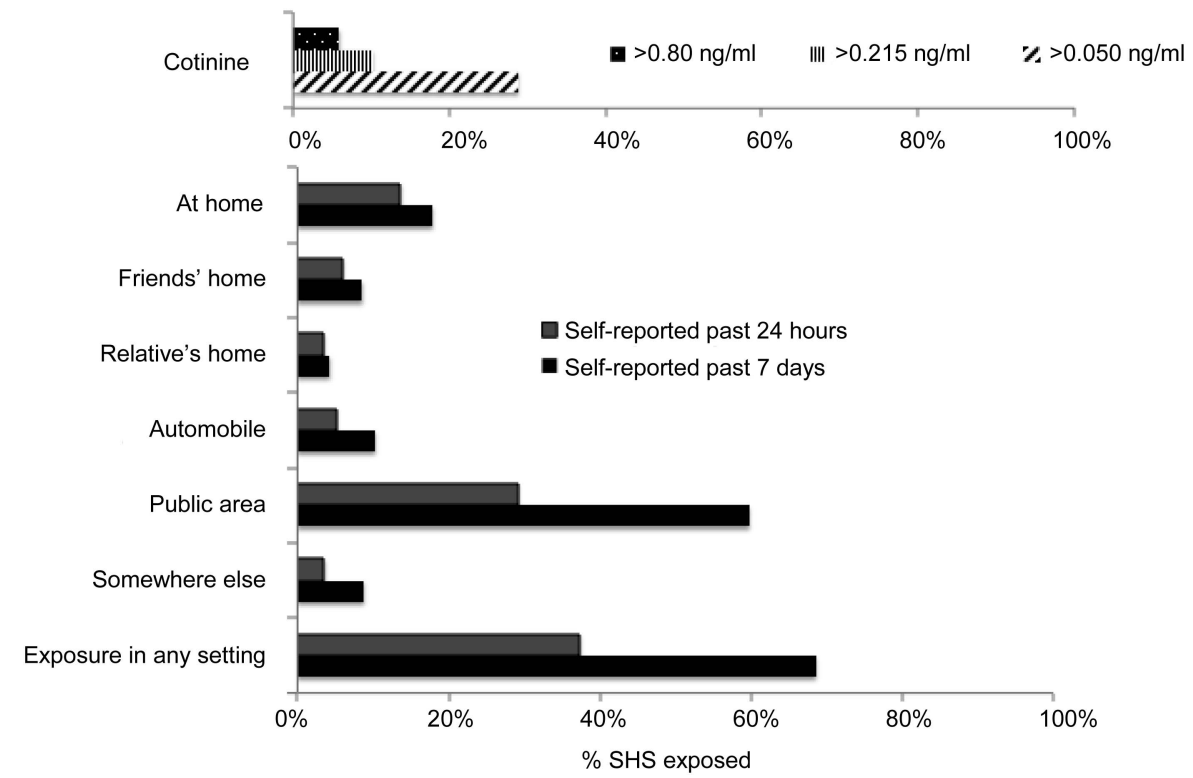

Figure 2 Per cent of sample with recent secondhand smoke (SHS) exposure based on serum cotinine and self-report items.

multi-item scales. For classification analysis, sum scores exceeding one on the multi-item scales, indicating SHS exposure in at least one of the settings assessed, were coded as exposed (1) versus unexposed (0). For cotinine cut-off points of 0.215 and $0.80 \mathrm{ng} / \mathrm{mL}$, the measure that maximised correct classification $(>85 \%)$ with the best balance of sensitivity $(\geq 75 \%)$ and specificity (>85\%) was the 7-day assessment of SHS exposure in one's own home. This single item measure outperformed the multi-item scales and the item assessing home smoking rules and had better sensitivity than the past 24-h assessment of SHS exposure in one's home. At the lowest cotinine cut-point of $0.05 \mathrm{ng} / \mathrm{mL}$, sensitivity was low for all of the measures $(<45 \%)$, while specificity was high (80\%-99\%). Correct classification was $\geq 70 \%$. At this level, the item assessing past 7-day SHS exposure in one's own home also provided the best balance with regard to sensitivity and specificity, with correct classification of $75 \%$.

A non-parametric test of the distribution of cotinine levels by participant reports of SHS exposure in the home in the past 7 days indicated a significant group difference, $\mathrm{p}<0.001$. The median cotinine value was 0 (IQR $0-0.04$ ) for those who denied and $0.14 \mathrm{ng} / \mathrm{mL}$ (IQR 0-1.14) for those who reported past 7-day SHS exposure in their home.

\section{DISCUSSION}

Evaluated among cardiology patients, our findings support the validity of self-reported recent SHS exposure. According to a 2013 review of SHS measures, ours is the first study to evaluate the validity of self-reported assessments of adult SHS exposure at home, in transport and in social situations (ie, homes of friends and family). ${ }^{19}$ Given the increased restrictions on SHS in worksites, restaurants, bars, parks and other public places, measures are needed to detect exposure in remaining locations, which are largely private residential settings and automobiles not directly impacted by most governmental and organisational policies.

Based on the current findings, the measure that optimised sensitivity with good specificity and correct classification was the single item assessment of exposure in the past 7 days to tobacco smoke in one's own residence (ASHES-7d Home). The ASHES-7d Home item demonstrated consistently good validity across all three cotinine cut-off points used to indicate significant SHS exposure. Reported SHS exposure in one's own home appears to be a clinical indicator of elevated levels of exposure and suggests the need for intervention to convey the importance of limiting SHS exposure for cardiovascular health. Of note, actual reported exposure to SHS in one's own home was a more valid indicator than a more general assessment of home smoking rules, suggesting some visitors to patients' homes may not be respecting patients' smoking bans or smoking by near neighbours may be having exposure effects.

Assessments of SHS exposure in other residential settings (ie, homes of family and friends) and automobiles (specifically in the past $24 \mathrm{~h}$ ) also correlated significantly with cotinine, but occurred less commonly and had poorer accuracy with classification. Nevertheless, the items demonstrated evidence of validly assessing SHS exposure in these specific settings. SHS exposure in public or other settings was reported by most participants,

Table 1 Point-biserial correlations of cotinine with self-report secondhand smoke (SHS) exposure measures

\begin{tabular}{lllllllll}
\hline & Personal residence & Friends' residence & Relatives' residence & Automobile & Public place & Other area & 4-Item sum scale & 3-Item sum scale \\
\hline $24 \mathrm{~h}$ & $0.37^{*}$ & $0.28^{*}$ & $0.43^{*}$ & $0.26^{*}$ & 0.05 & -0.02 & $0.46^{*}$ & - \\
7 days & $0.41^{*}$ & $0.45^{*}$ & $0.38^{*}$ & 0.16 & 0.01 & -0.05 & - & $0.53^{*}$ \\
\hline
\end{tabular}

Scales calculated for items with significant correlations: the 3-item scale sums past 7-day SHS exposure in the residence of one's own, of friends and of relatives, range 0-3; the 4-item scale sums past 24-h residential SHS exposure and automobile SHS exposure, range $0-4$. ${ }^{*} \mathrm{p}<0.01$. 
Table 2 Sensitivity, specificity and correct classification rates (\%) of measures of secondhand smoke exposure

\begin{tabular}{|c|c|c|c|c|c|c|c|c|c|c|}
\hline & \multicolumn{2}{|c|}{ Own home } & \multicolumn{2}{|c|}{$\begin{array}{l}\text { Relative's } \\
\text { home }\end{array}$} & \multicolumn{2}{|c|}{ Friend's home } & \multirow{2}{*}{$\begin{array}{l}\text { Automobile } \\
24 \mathrm{~h}\end{array}$} & \multirow{2}{*}{$\begin{array}{l}\text { 4-Item scale } \\
24 \mathrm{~h}\end{array}$} & \multirow{2}{*}{$\begin{array}{l}3 \text {-Item scale } \\
7 \text { days }\end{array}$} & \multirow[b]{2}{*}{ Home smoking rule } \\
\hline & $24 \mathrm{~h}$ & 7 days & $24 \mathrm{~h}$ & 7 days & $24 \mathrm{~h}$ & 7 days & & & & \\
\hline \multicolumn{11}{|l|}{$>0.05 \mathrm{ng} / \mathrm{mL}$} \\
\hline Sensitivity & 32.4 & 38.2 & 5.9 & 9.1 & 11.8 & 21.2 & 14.7 & 35.3 & 41.2 & 44.1 \\
\hline Specificity & 94.0 & 90.5 & 97.6 & 97.6 & 96.4 & 96.4 & 98.8 & 90.5 & 86.9 & 79.8 \\
\hline Correct classification & 76.2 & 75.4 & 71.2 & 72.4 & 72.0 & 75.0 & 74.5 & 74.6 & 73.8 & 69.5 \\
\hline \multicolumn{11}{|l|}{$>0.215 \mathrm{ng} / \mathrm{mL}$} \\
\hline Sensitivity & 66.7 & 75.0 & 16.7 & 16.7 & 33.3 & 41.7 & 41.7 & 75.0 & 75.0 & 66.7 \\
\hline Specificity & 92.5 & 88.7 & 98.1 & 97.1 & 97.2 & 95.2 & 99.1 & 89.6 & 84.9 & 77.4 \\
\hline Correct classification & 89.9 & 87.3 & 89.8 & 88.8 & 90.7 & 89.8 & 93.2 & 88.1 & 83.9 & 76.3 \\
\hline \multicolumn{11}{|l|}{$>0.80 \mathrm{ng} / \mathrm{mL}$} \\
\hline Sensitivity & 71.4 & 85.7 & 28.6 & 28.6 & 28.6 & 57.1 & 42.9 & 71.4 & 85.7 & 71.4 \\
\hline Specificity & 90.1 & 86.5 & 98.2 & 97.2 & 95.5 & 94.5 & 97.3 & 86.5 & 82.9 & 75.7 \\
\hline Correct classification & 88.9 & 86.5 & 94.1 & 93.1 & 91.5 & 92.2 & 94.0 & 85.6 & 83.1 & 75.6 \\
\hline
\end{tabular}

yet demonstrated weak association with measured cotinine levels, suggesting the exposures were of brief duration and/or of low concentration. Cotinine has a $72 \mathrm{~h}$ window of detection, so it is possible that more participants were, in fact, SHS-exposed in the past week, but not detected given the relatively short halflife of the biomarker. Future research is needed to evaluate the items in relation to NNAL, which has a longer half-life, and would likely yield higher estimates of exposure. Replication also is needed in a larger sample with appropriate diversity to permit subgroup analyses (eg, by gender, age, ethnicity) to determine if the single item of past week exposure in one's residence is the optimal SHS assessment. Notably, in the current sample, correlations between cotinine and the ASHES-7d Home item were comparable for men and women ( $\mathrm{r}$ values $>0.40$ and $\mathrm{p}$ values $<0.01$ ); determination of correct classification rates by gender, however, was not possible due to expected cell counts $<5$ in the $\chi^{2}$ analyses. The prevalence of SHS exposure in the current sample (mean age $=63$ ), while relatively low-ranging from $6 \%$ (cotinine $>0.80 \mathrm{ng} / \mathrm{mL}$ ) to $29 \%$ (cotinine $>0.05$ )approximates SHS levels reported for non-smoking older adults nationally. ${ }^{10}$ According to data from the National Health and Nutrition Examination Survey, 37\% of adults had cotinine levels $>0.05$ in 2007-2008, with lower exposure among older adults (31.6\% among adults 60 years and older) and a clear trend of decreasing SHS exposure over time. The findings for sensitivity and specificity may not generalise to samples with greater SHS exposure.

SHS surveillance has been in place for over 20 years; ${ }^{24} 25$ yet, among adult patients, SHS has received little attention in clinical practice. In contrast, identification and reduction of primary tobacco use is one of the strongest public health prevention and medical treatment recommendations. ${ }^{11}$ A recent comparative review concluded that a number of blood biomarkers ordered routinely in cardiology have weak evidence of predictive effects for cardiovascular disease. ${ }^{26}$ SHS exposure's harmful cardiovascular effects are established ${ }^{2}$ and warrant the adoption of SHS screening practices in clinical care. Brief and simple to administer, the ASHES-7d Home single item screener offers a practical and cost-effective means to identify patients at risk and in need of provider counselling to avoid SHS exposure for protecting their health. The current findings support the use of the
ASHES-7d Home screener to detect SHS exposure, easily incorporated into assessment of other major vital signs in cardiology.

\section{What this paper adds}

Evaluated among cardiology patients, our findings support the validity of self-reported recent secondhand smoke (SHS) exposure. According to a 2013 review of SHS measures, this is the first study to evaluate the validity of self-reported assessments of adult SHS exposure at home, in transport and in social situations (ie, homes of friends and family). The single item adult secondhand exposure to smoke-7d (ASHES-7d) Home screener is brief, assesses recent SHS exposure over a week's time, and yielded the optimal balance of sensitivity and specificity. While further investigation is warranted, the current findings support the use of the ASHES-7d Home screener to detect SHS exposure and can be easily incorporated into assessment of other major vital signs in cardiology.

Acknowledgements We greatly appreciate the contributions of Nora Cordero, the study phlebotomist, and Chanel Garcia for collection of patient data. We thank Kevin Delucchi, PhD, for his biostatistical expertise and consultation on the study analyses.

Contributors JJP conceived of and led the conduct of this study including study design, recruitment and data collection, analyses and writing up study findings. WG's funding from FAMRI supported the research. WG participated in study design, facilitated recruitment at the clinical sites and provided input into the written manuscript. KCY-W contributed to the writing up of study findings. NB contributed to study design, facilitated participant recruitment, oversaw laboratory testing of obtained samples, and provided key input into the interpretation and write up of study findings.

Funding The Flight Attendant Medical Research Institute, Miami, Florida, USA; the National Heart Lung and Blood Institute (\#T32 HL007034), the National Institute of Mental Health (\#R01 MH083684), and the National Institute on Drug Abuse (\#P50 DA09253), Bethesda, Maryland, USA; and the State of California Tobacco-Related Disease Research Program (\#21BT-0018), Oakland, California, USA

Competing interests Unrelated to the research presented here, Dr Prochaska is an ad hoc advisory board member, grant reviewer and principal investigator on an investigator initiated research award with Pfizer Inc. Dr Benowitz is a paid consultant to pharmaceutical companies that market smoking-cessation medications, 
including Pfizer, GlaxoSmithKline and McNeil, and has served as a paid expert witness in litigation again tobacco companies.

\section{Ethics approval UCSF.}

Provenance and peer review Not commissioned; externally peer reviewed.

Data sharing statement The unpublished data from the study are held by Dr Prochaska at Stanford University and Dr Grossman at the University of California San Francisco. The data are available for collaborative efforts upon request.

\section{REFERENCES}

1 U.S. Department of Health and Human Services. The health consequences of involuntary exposure to tobacco smoke: a report of the surgeon general. 2006 http://www.surgeongeneral.gov/library/reports/secondhandsmoke/report-index.htm (accessed 20 Oct 2012).

2 Committee on Secondhand Smoke Exposure and Acute Coronary Events, Institute of Medicine. Secondhand smoke exposure and cardiovascular effects: making sense of the evidence, committee on secondhand smoke exposure and acute coronary events. 2009. http://www.iom.edu/Reports/2009/Secondhand-Smoke-Exposure-and Cardiovascular-Effects-Making-Sense-of-the-Evidence.aspx (accessed 20 Oct 2012)

3 Erhardt L. Cigarette smoking: an undertreated risk factor for cardiovascular disease. Atherosclerosis 2009;205:23-32.

4 Callinan JE, Clarke A, Doherty $K$, et al. Legislative smoking bans for reducing secondhand smoke exposure, smoking prevalence and tobacco consumption. Cochrane Database Syst Rev 2010;(4):CD005992.

5 Haw SJ, Gruer L. Changes in exposure of adult non-smokers to secondhand smoke after implementation of smoke-free legislation in Scotland: national cross sectional survey. BMJ 2007;335:549.

6 IARC. Evaluating the effectiveness of smoke-free policies. IARC Handb Cancer Prev 2009;13:1-348.

7 Lightwood JM, Glantz SA. Declines in acute myocardial infarction after smoke-free laws and individual risk attributable to secondhand smoke. Circulation 2009;120:1373-9.

8 Meyers DG, Neuberger JS, He J. Cardiovascular effect of bans on smoking in public places: a systematic review and meta-analysis. J Am Coll Cardiol 2009;54:1249-55.

9 Hurt RD, Weston SA, Ebbert J0, et al. Myocardial infarction and sudden cardiac death in Olmsted county, Minnesota, before and after smoke-free workplace laws. JAMA Intern Med 2012;172:1635-41.

10 Centers for Disease Control and Prevention. Vital signs: nonsmokers' exposure to secondhand smoke: United States, 1999-2008. MMWR Morb Mortal Wkly Rep 2010;59:1141-6.
11 Tobacco Use and Dependence Guideline Panel. Treating tobacco use and dependence: 2008 update. 2008. http://www.ahrq.gov/clinic/tobacco/treating_ tobacco_use08.pdf (accessed 20 Oct 2012).

12 Benowitz NL. Cotinine as a biomarker of environmental tobacco smoke exposure. Epidemiol Rev 1996;18:188-204.

13 Avila-Tang E, Al-Delaimy WK, Ashley DL, et al. Assessing secondhand smoke using biological markers. Tob Control 2013;22:164-71.

14 Bernert JT, Pirkle JL, Xia Y, et al. Urine concentrations of a tobacco-specific nitrosamine carcinogen in the U.S. population from secondhand smoke exposure. Cancer Epidemiol Biomarkers Prev 2010;19:2969-77.

15 Sleiman M, Gundel LA, Pankow JF, et al. Formation of carcinogens indoors by surface-mediated reactions of nicotine with nitrous acid, leading to potential thirdhand smoke hazards. Proc Natl Acad Sci USA 2010;107:6576-81.

16 Benowitz NL, Schultz KE, Haller CA, et al. Prevalence of smoking assessed biochemically in an urban public hospital: a rationale for routine cotinine screening. Am J Epidemiol 2009;170:885-91.

17 Venn A, Britton J. Exposure to secondhand smoke and biomarkers of cardiovascular disease risk in never-smoking adults. Circulation 2007;115:990-5.

18 Whincup PH, Gilg JA, Emberson JR, et al. Passive smoking and risk of coronary heart disease and stroke: prospective study with cotinine measurement. BMJ 2004;329:200-5

19 Avila-Tang E, Elf JL, Cummings KM, et al. Assessing secondhand smoke exposure with reported measures. Tob Control 2013;22:156-63.

20 Chen R, Tavendale R, Tunstall-Pedoe H. Measurement of passive smoking in adults: self-reported questionnaire or serum cotinine? J Cancer Epidemiol Prev 2002;7:85-95.

21 SRNT Subcommittee on Biochemical Verification. Biochemical verification of tobacco use and cessation. Nicotine Tob Res 2002:4:149-59.

22 Jacob P III, Yu L, Duan M, et al. Determination of the nicotine metabolites cotinine and trans-3'-hydroxycotinine in biologic fluids of smokers and non-smokers using liquid chromatography-tandem mass spectrometry: biomarkers for tobacco smoke exposure and for phenotyping cytochrome P450 2a6 activity. J Chromatogr B Analyt Technol Biomed Life Sci 2011:879:267-76.

23 Jarvis MJ, Tunstall-Pedoe $\mathrm{H}$, Feyerabend $\mathrm{C}$, et al. Comparison of tests used to distinguish smokers from nonsmokers. Am J Public Health 1987:77:1435-8.

24 Hirayama T. Non-smoking wives of heavy smokers have a higher risk of lung cancer: a study from japan. BMJ 1981;282:183-5.

25 Pirkle JL, Flegal KM, Bernert JT, et al. Exposure of the us population to environmental tobacco smoke: the third national health and nutrition examination survey, 1988 to 1991. JAMA 1996;275:1233-40.

26 Ioannidis JP, Tzoulaki I. Minimal and null predictive effects for the most popular blood biomarkers of cardiovascular disease. Circ Res 2012;110:658-62. 